\title{
Interlayer exchange coupling in ordered Fe nanocluster arrays grown on $\mathrm{Al}_{2} \mathrm{O}_{3} / \mathrm{Ni}_{3} \mathrm{Al}(111)$
}

\author{
S. Vlaic, ${ }^{1, *}$ L. Gragnaniello,,${ }^{1 \dagger}$ S. Rusponi, ${ }^{1}$ A. Cavallin,,${ }^{1, \ddagger}$ F. Donati, ${ }^{1}$ Q. Dubout, ${ }^{1}$ C. Piamonteze,,${ }^{2}$ J. Dreiser, ${ }^{2}$ \\ F. Nolting, ${ }^{2}$ and H. Brune ${ }^{1, \S}$ \\ ${ }^{1}$ Institute of Condensed Matter Physics, École Polytechnique Fédérale de Lausanne, CH-1015 Lausanne, Switzerland \\ ${ }^{2}$ Swiss Light Source, Paul Scherrer Institut, CH-5232 Villigen PSI, Switzerland \\ (Received 21 February 2014; revised manuscript received 13 May 2014; published 3 June 2014)
}

\begin{abstract}
We have combined magneto-optical Kerr effect, scanning tunneling microscopy, and x-ray magnetic circular dichroism to study the magnetic properties and the morphology of Fe nanoparticles grown on 2 ML thick $\mathrm{Al}_{2} \mathrm{O}_{3} / \mathrm{Ni}_{3} \mathrm{Al}(111)-(\sqrt{67} \times \sqrt{67}) \mathrm{R} 12.2^{\circ}$ with and without $\mathrm{Pd}$ seeding. The $\mathrm{Ni}_{3} \mathrm{Al}(111)$ substrate is ferromagnetic and shows two transition temperatures. The first, $T_{\mathrm{C}_{1}}=81 \pm 3 \mathrm{~K}$, is attributed to a $20-30 \mathrm{~nm}$ thick slightly $\mathrm{Ni}$ enriched region; the second, $T_{\mathrm{C}_{2}}=240 \pm 12 \mathrm{~K}$, is attributed to a much thinner and more strongly $\mathrm{Ni}$ enriched near interface region that contains $\mathrm{Ni}$ clusters embedded in the alloy matrix. The magnetic properties of the Fe cluster superlattice are strongly influenced by the superexchange coupling between Fe clusters and the underlying Ni clusters in that near interface region. Since the Ni clusters are at different distances from the oxide/metal interface, this coupling oscillates between ferro- and antiferromagnetic such that the overall magnetic moment is not increased by the Fe clusters. Pd seeding does not influence the magnetic properties of the system. The intrinsic Fe cluster properties, such as Curie temperature and easy magnetization axis, are accessed for $T>T_{\mathrm{C}_{2}}$. We find out-of-plane easy magnetization axes and $T_{\mathrm{C}} \approx 300 \mathrm{~K}$ for cluster sizes above 440 atoms.
\end{abstract}

DOI: 10.1103/PhysRevB.89.245402

PACS number(s): 75.75.-c, 78.70.Dm, 75.70.-i

\section{INTRODUCTION}

The bottom-up fabrication of ordered two-dimensional (2D) arrays of nanoparticles with narrow size distributions $[1,2]$ enables the study of their catalytic and magnetic properties as a function of size and composition with spatially integrating techniques. Very small transition metal nanoparticles can exhibit a strong enhancement of these properties with respect to their bulk counterparts, due to the large number of low-coordinated atoms [3-6]. For heterogeneous catalysis and future magnetic storage applications it is crucial to identify the minimum cluster size as morphologically stable and having the desired catalytic or magnetic properties. Among the possible approaches to the fabrication of well-ordered 2D arrays, referred to as 2D superlattices, kinetically controlled growth on template surfaces, created either by strain relief patterns or surface reconstructions, emerged as the most promising [2,7-13].

The self-limiting two monolayer thick alumina film grown by high temperature oxidation of $\mathrm{Ni}_{3} \mathrm{Al}(111)$ has excellent template properties. Its $(\sqrt{67} \times \sqrt{67}) \mathrm{R} 12.2^{\circ}$ unit cell has corner holes going down to the metal substrate [14]. They form a hexagonal lattice with a $4.1 \mathrm{~nm}$ lattice constant. They can be filled by $\mathrm{Pd}$ atoms deposited at $300 \mathrm{~K}$ creating very well ordered Pd cluster superlattices [15]. Amongst the transition metal elements investigated so far, $\mathrm{Pd}$ is the only one diffusing

\footnotetext{
*Present address: CNRS, Institute NEEL, F-38042 Grenoble, France and Univ. Grenoble Alpes, Inst NEEL, F-38042 Grenoble, France.

${ }^{\dagger}$ Present address: Fachbereich Physik, Universität Konstanz, 78457 Konstanz, Germany.

${ }^{\ddagger}$ Present address: Max-Planck-Institut für Mikrostrukturphysik, Weinberg 2, D-06120, Halle/Saale, Germany.

${ }^{\S}$ Corresponding author: harald.brune@epfl.ch
}

into the corner holes $[14,16]$. Therefore it has been used as seed to nucleate other transition metal elements leading to well ordered arrays of quasibimetallic nanoparticles of AuPd [17], FePd and CoPd [14,18], and NiPd [19].

It has been suggested that such nanostructures on alumina/ $\mathrm{Ni}_{3} \mathrm{Al}(111)$, when made of ferromagnetic materials, can be of special interest as a model system for potential magnetic applications [18] since the uniform size of the nanoparticles could result in well-defined and homogeneous magnetic properties [20,21]. Nevertheless it has to be noticed that the substrate magnetism can also play a role, influencing the magnetic behavior of the nanoparticles. In fact, $\mathrm{Ni}_{3} \mathrm{Al}(111)$ is a weak itinerant ferromagnet whose magnetic properties strongly depend on its exact chemical composition [22-24]. Moreover the high temperature oxidation of the surface, through which the ultrathin $\mathrm{Al}_{2} \mathrm{O}_{3}$ layer is formed, involves an increase of the $\mathrm{Ni}$ concentration below the surface due to the Al incorporation into the oxide [25]. Thus, magnetic nanoparticles on alumina/ $\mathrm{Ni}_{3} \mathrm{Al}(111)$ can even be seen as a model system for spin valves where one of the electrodes has nanometric dimensions.

Despite many recent efforts to reveal the structure of the surface layers of $\mathrm{Al}_{2} \mathrm{O}_{3} / \mathrm{Ni}_{3} \mathrm{Al}(111)$ [14,19,26], an accurate characterization of the composition of those layers interfacing the oxide from below, and of the resulting magnetic properties, is still lacking. Here we report a detailed investigation of the magnetic properties of the pristine substrate before and after the growth of alumina and finally after the growth of $\mathrm{Fe}$ nanoparticles on top. We find that the magnetism of this system is strongly influenced by an inhomogeneous stoichiometry of the alloy substrate across the layers interfacing the oxide leading to an exchangelike coupling between $\mathrm{Fe}$ nanoparticles and substrate. The magnetic properties of the Fe clusters do not depend on whether they are seeded on $\mathrm{Pd}$ or not. 


\section{EXPERIMENTAL DETAILS}

The $\mathrm{Ni}_{3} \mathrm{Al}$ single crystal was cleaned by $\mathrm{Ar}^{+}$-ion bombardment $(T=600 \mathrm{~K}, E=2 \mathrm{keV}, I=0.4 \mu \mathrm{A}$ for our $7 \mathrm{~mm}$ diameter sample, $t=40 \mathrm{~min}$ ) followed by two annealing cycles at 1150 and $1050 \mathrm{~K}$, each for 10 minutes. The selflimiting $2 \mathrm{ML}$ thick alumina was prepared by oxygen exposure $\left(p_{O_{2}}=5 \times 10^{-8}\right.$ mbar) of the clean $\mathrm{Ni}_{3} \mathrm{Al}(111)$ surface at $1050 \mathrm{~K}$ for one hour. After stopping the $\mathrm{O}_{2}$ dosage, the crystal was kept for one more hour at this temperature. Fe and $\mathrm{Pd}$ were deposited at $300 \mathrm{~K}$ by atomic beam epitaxy, $\mathrm{Fe}$ from a high-purity $(99.995 \%)$ rod using a commercial $e$-beam evaporator, and $\mathrm{Pd}$ from a high-purity $\mathrm{Pd}$ filament by resistive heating. The deposition flux, determined by scanning tunneling microscopy (STM) on the clean $\mathrm{Ni}_{3} \mathrm{Al}(111)$ surface where the growth is layer by layer, was 0.3 and $0.19 \mathrm{ML} / \mathrm{min}$ for Fe and $\mathrm{Pd}$, respectively. One monolayer (ML) is defined as one $\mathrm{Fe}(\mathrm{Pd})$ atom per $\mathrm{Ni}_{3} \mathrm{Al}(111)$ substrate atom, i.e., as four atoms per $p(2 \times 2)$ unit cell of the $\mathrm{Ni}_{3} \mathrm{Al}(111)$ surface $[27,28]$.

The morphology of $\mathrm{Fe}$ clusters on $\mathrm{Al}_{2} \mathrm{O}_{3} / \mathrm{Ni}_{3} \mathrm{Al}(111)$ was investigated by STM and their magnetic properties by magneto-optical Kerr effect (MOKE), X-ray absorption spectroscopy (XAS), and X-ray magnetic circular dichroism (XMCD). STM and MOKE were carried out in an ultrahigh vacuum (UHV) chamber at École Polytechnique Fédérale de Lausanne (EPFL) [29,30]. XAS and XMCD measurements were performed at our EPFL-PSI beamline X-Treme at Swiss Light Source (SLS) [31].

The light source used for MOKE measurements is a diode laser of wavelength equal to $785 \mathrm{~nm}$. MOKE zero-field susceptibility $(\chi)$ measurements were performed in polar geometry with a sinusoidal magnetic field of $4 \mathrm{mT}$ amplitude and $11 \mathrm{~Hz}$ frequency. Magnetization curves $M(H)$ were acquired in transverse and polar geometry with sweep rates of $0.7 \mathrm{mT} / \mathrm{s}$ and $160 \mathrm{mT} / \mathrm{s}$, respectively. In order to compare Kerr signals from the bare and two ML oxide covered $\mathrm{Ni}_{3} \mathrm{Al}$ substrate and from samples with the Fe clusters on top, we normalized the Kerr intensities considering that the polar Kerr signal, for small rotation angles, can be written as [32,33]:

$$
\frac{\Delta I}{I(0)} \approx \frac{2 \theta \phi}{\phi^{2}+\gamma},
$$

where $I(0)$ is the zero-field intensity of the reflected light measured after the analyzer, $\Delta I=I(H)-I(0)$ the variation of Kerr signal produced by the external field, $\theta$ the Kerr rotation angle, $\phi$ labels the angle of the analyzer axis with respect to maximum extinction, and $\gamma$ the depolarization fraction taking into account the polarizer efficiency and the range of angles of incidence in the focused beam on the sample. In our setup $\phi$ is set to $0.5^{\circ}$ to optimize the Kerr efficiency [33], and the typical value for $\gamma$ is $2 \times 10^{-5}$. Equation (1) holds also for polar magnetization curves where the Kerr rotation angle can be derived. In addition to the sample composition, the Kerr intensity also depends on the optical surface quality [34] and it varies between sample preparation cycles since this requires the realignment of the MOKE setup to optimize its signal. The observed variations in the $\chi$ intensities and $M(H)$ curves were $2 \%$ over a given sample and $15 \%$ between preparation cycles. These are systematic variations that can't be eliminated by the above normalization procedure.
The STM measurements were performed at $300 \mathrm{~K}$ in constant current mode using a home-built variable-temperature STM equipped with an electrochemically etched $\mathrm{W}$ tip. The XAS and XMCD measurements were performed in the total electron yield (TEY) mode and with the X-ray beam and the magnetic field aligned parallel and forming an angle $\theta$ with the surface normal. The out-of-plane and close to in-plane XMCD signals were recorded at $\theta=0^{\circ}$ and $\theta=70^{\circ}$, respectively. The XMCD spectra were recorded at a field of $1 \mathrm{~T}$ saturating the samples for both field orientations. Ni magnetization curves were acquired using the peak of the $\mathrm{L}_{3} \mathrm{XMCD}$ intensity at $849 \mathrm{eV}$ divided by the pre-edge intensity at $845 \mathrm{eV}$.

\section{RESULTS AND DISCUSSION}

Since the substrate is magnetic, and MOKE not element specific, it measures the superposition of substrate and $\mathrm{Fe}$ cluster magnetism. We therefore first characterize the substrate magnetism before turning to the magnetic properties of the $\mathrm{Fe}$ cluster superlattice.

\section{A. Substrate magnetism and Ni surface enrichment}

The $2 \mathrm{ML}$ thick alumina layer on $\mathrm{Ni}_{3} \mathrm{Al}(111)$ forms a $(\sqrt{67} \times \sqrt{67}) \mathrm{R} 12.2^{\circ}$ unit cell with respect to the $p(2 \times 2)$ unit cell of the $\mathrm{Ni}_{3} \mathrm{Al}(111)$ surface with its $505 \mathrm{pm}$ long unit vectors $[27,28]$. The stacking and stoichiometry of the two $\mathrm{Al}_{2} \mathrm{O}_{3}$ layers, and the existence of corner holes in the top layer, have been revealed from a combination of STM and density functional theory (DFT) [14]. Depending on tunnel voltage $V_{\mathrm{t}}$, this surface appears to the STM either as a honeycomb network or as a hexagonal lattice of protrusions [18,27]. An STM image of our sample taken in the first imaging mode is shown in Fig. 1(a) and reveals the long-range ordered superstructure.

Figure 1(b) compares the temperature-dependent out-ofplane zero-field susceptibilities of the clean alloy surface (red) with the alumina covered one (black). Both signals are very similar and present two plateaus with transition temperatures $T_{\mathrm{C}_{1}}=79 \pm 1 \mathrm{~K}$ and $T_{\mathrm{C}_{2}}=236 \pm 3 \mathrm{~K}$. The XMCD Ni magnetization curves of Fig. 1(c) reveal an in-plane easy axis for $\mathrm{Al}_{2} \mathrm{O}_{3} / \mathrm{Ni}_{3} \mathrm{Al}(111)$ at $160 \mathrm{~K}$. Therefore $\chi(T)$ in Fig. 1(b) probes the hard-axis component of $\mathbf{M}$ generated by the torque of the small oscillating out-of-plane field. This signal depends on the ratio of $M$ and the magnetic anisotropy energy $K$, and is thus to first order independent of $T$ leading to the observed plateau. The plateau ends at the Curie temperature $T_{\mathrm{C}}$ where the magnitude of $\mathbf{M}$ strongly decreases. The fact that we see two plateaus signifies that we have two coexisting magnetic systems, each with its phase transition.

The coexistence of two such systems has been suggested for the surface of a $\mathrm{Ni}_{3+\delta} \mathrm{Al}_{1-\delta}$ bulk alloy with $\delta=0.084$ that exhibited, as in the present case, two Curie temperatures, both very close to our values [24]. The first has been attributed to the weak itinerant ferromagnetism of the $\mathrm{Ni}_{3} \mathrm{Al}$ bulk alloy [22,23] and the second to Ni clusters embedded into this alloy [24].

The Curie temperature of $\mathrm{Ni}_{3} \mathrm{Al}$ bulk strongly depends on stoichiometry. While the stoichiometric alloy has $T_{\mathrm{C}}=$ $40 \mathrm{~K}$, already $2 \%$ higher $\mathrm{Ni}$ content almost doubles this value [22,23]. The value found for $\mathrm{Ni}_{3+\delta} \mathrm{Al}_{1-\delta}$ with $\delta=0.084$ is within the error bar identical with our $T_{\mathrm{C}_{1}}$ [24]. We thus 
(a)

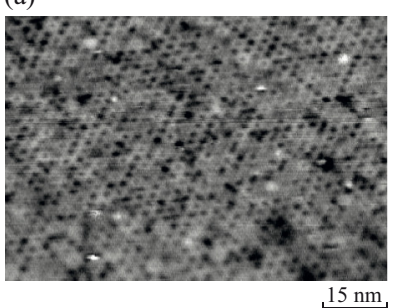

$15 \mathrm{~nm}$

(c)
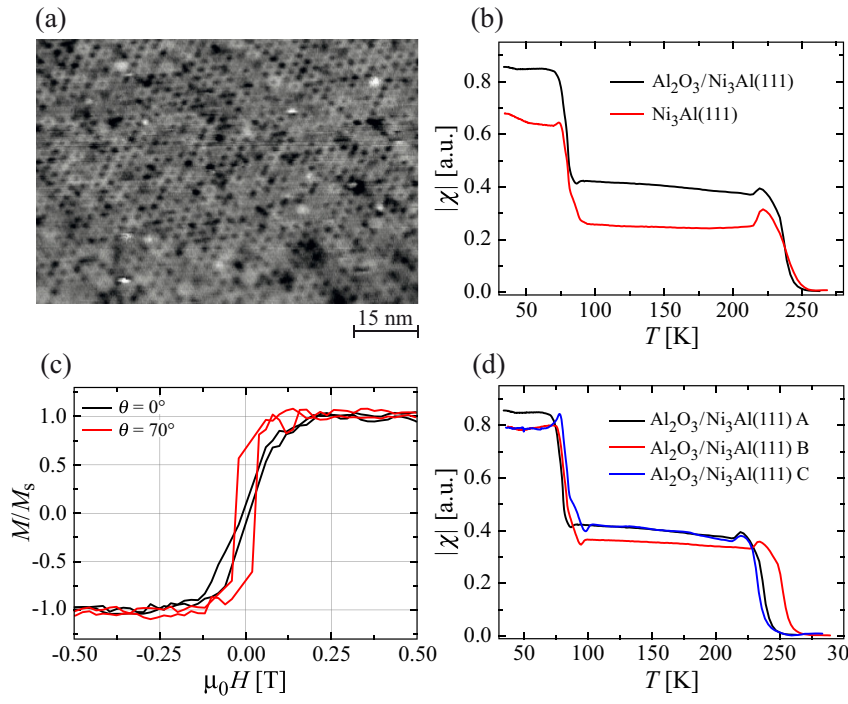

(d)

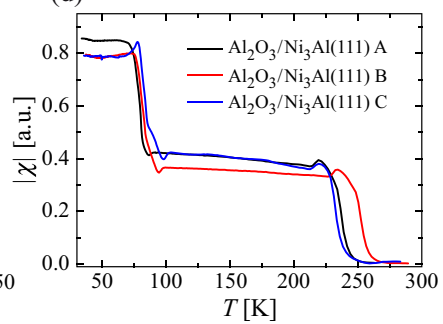

FIG. 1. (Color online) (a) STM image of the $\mathrm{Al}_{2} \mathrm{O}_{3}$ bilayer on $\mathrm{Ni}_{3} \mathrm{Al}(111)\left(V_{\mathrm{t}}=3.5 \mathrm{~V}, I_{\mathrm{t}}=0.1 \mathrm{nA}\right)$. (b) Out-of-plane zero field magnetic susceptibility $\chi(T)$ of pristine $\mathrm{Ni}_{3} \mathrm{Al}(111)$ (red) and $\mathrm{Al}_{2} \mathrm{O}_{3} / \mathrm{Ni}_{3} \mathrm{Al}(111)$ (black). The transitions between the plateaus are at $T_{\mathrm{C}_{1}}=79 \pm 1 \mathrm{~K}$ and $T_{\mathrm{C}_{2}}=236 \pm 3 \mathrm{~K}$. (c) XMCD Ni $M(H)$ curves recorded on $\mathrm{Al}_{2} \mathrm{O}_{3} / \mathrm{Ni}_{3} \mathrm{Al}(111)$ at $T=160 \mathrm{~K}$ at normal (black) and grazing incidence (red) showing an in-plane easy axis. (d) Influence of many sputter-annealing-oxidation cycles. $\mathrm{B}$ and $\mathrm{C}$ have been acquired two and four months after $\mathrm{A}$, respectively. $T_{\mathrm{C}_{1}}$ increases monotonically while $T_{\mathrm{C}_{2}}$ evolves in a nonmonotonic way.

attribute the first magnetic phase transition of our substrate to a slightly $\mathrm{Ni}$ enriched near surface region of the crystal that is at least as thick as the MOKE probing depth of 20-30 nm $[35,36]$.

The second plateau with much higher $T_{\mathrm{C}}$ either stems from a very thin and strongly $\mathrm{Ni}$ enriched layer located directly at the $\mathrm{Ni}_{3} \mathrm{Al} /$ vacuum, respectively, $\mathrm{Ni}_{3} \mathrm{Al} / \mathrm{Al}_{2} \mathrm{O}_{3}$ interface, or from pure Ni clusters embedded into the alloy and distributed over the MOKE probing depth [24]. Although the first interpretation is supported by $T_{\mathrm{C}_{2}}$ being close to that of a $2 \mathrm{ML} \mathrm{Ni}$ thin film on $\mathrm{Cu}(111)$ [37], we prefer the second, proposed in Ref. [24]. As we will see below it allows us to explain the nontrivial observed magnetic coupling of the Fe clusters across the oxide to the substrate.

The $\mathrm{Ni}$ enhancement in the near surface region can be caused by $\mathrm{Al}$ segregation upon annealing, reducing the $\mathrm{Al}$ concentration in a certain thickness below the surface [38]. In addition, $\mathrm{Al}$ has a slightly higher sputtering yield with respect to $\mathrm{Ni}[39,40]$ and is therefore depleted at each preparation cycle. Finally, the initial stoichiometry of the $\mathrm{Ni}_{3} \mathrm{Al}$ crystal might have been slightly unbalanced.

Further evidence of Ni enrichment in the near surface region is given by the evolution of both $T_{\mathrm{C}}$ 's over many surface preparation and oxidation cycles. Figure 1(d) presents $\chi(T)$ of three freshly prepared oxide covered surfaces that span a time window of four months (named A, B, and C in temporal order). Very many preparation and oxidation cycles separate those measurements, since we prepared a fresh $\mathrm{Al}_{2} \mathrm{O}_{3} / \mathrm{Ni}_{3} \mathrm{Al}(111)$ surface almost every day. It is seen that $T_{\mathrm{C}_{1}}$ monotonically increases with time going from $79 \pm 1 \mathrm{~K}$ for $\mathrm{A}$ to $83 \pm 1 \mathrm{~K}$ for
$\mathrm{C}$, implying that the $\mathrm{Ni}$ concentration in the near surface region is slightly increasing with the number of preparation cycles. In contrast, $T_{\mathrm{C}_{2}}$ varies in a nonmonotonic way; it starts from $233 \pm 3 \mathrm{~K}$ for sample $\mathrm{A}$, goes to $251 \pm 3 \mathrm{~K}$ for $\mathrm{B}$, and back close to the initial value for $\mathrm{C}$. This oscillation is attributed to small changes in the embedded Ni cluster size or morphology, albeit the application of an unchanged preparation procedure.

We now turn to the $\chi$ intensities. While the differences observed in the height of the two plateaus in samples A, $\mathrm{B}$, and $\mathrm{C}$ are within the limit of our sensitivity, the overall increase from bare $\mathrm{Ni}_{3} \mathrm{Al}$ to $\mathrm{Al}_{2} \mathrm{O}_{3} / \mathrm{Ni}_{3} \mathrm{Al}$ in Fig. 1(b) is significant. This enhancement is evidence of a further increase of $\mathrm{Ni}$ content at the alloy/oxide interface caused by the depletion of $\mathrm{Al}$ incorporated into the oxide [25]. Increasing the Ni concentration directly below the oxide enhances $M$ and thus $\chi(T)$ recorded perpendicular to the easy axis. The almost unchanged $T_{\mathrm{C}_{2}}$ value in Fig. 1(b) suggests that the formation of the surface oxide does not modify the magnetic exchange energy of the embedded $\mathrm{Ni}$ clusters, while it probably increases the density and/or size of those located close to the $\mathrm{Al}_{2} \mathrm{O}_{3} / \mathrm{Ni}_{3} \mathrm{Al}$ interface.

\section{B. Equivalent Ni thickness at oxide/alloy interface}

We now estimate with XAS and XMCD the amount of $\mathrm{Ni}$ composing the Ni clusters, located close to the alloy/oxide interface, in terms of the thickness of an equivalent $\mathrm{Ni}$ film below the oxide layer. We note that the probing depth of XAS and XMCD in the TEY mode and for the electron energy of the Ni $L_{2,3}$ absorption edges is of the order of $2 \mathrm{~nm}$. The reported values vary between $1.4 \mathrm{~nm}[41,42]$ and $2.56 \pm 0.03 \mathrm{~nm}$ [43]. We consider the three subsequent layers, labeled $i=1$ for the $l=0.45 \mathrm{~nm}$ thick oxide [14,26], $i=2$ for the Ni clusters with equivalent layer thickness $d$ to be determined, and $i=3$ for the 20-30 nm thick near surface region with composition $\mathrm{Ni}_{3+\delta} \mathrm{Al}_{1-\delta}$, see Fig. 2(e).

We take advantage of the fact that the ferromagnetism of the $\mathrm{Ni}_{3+\delta} \mathrm{Al}_{1-\delta}$ near surface region can be turned on and off by measuring once below and once above $T_{\mathrm{C}_{1}}$. Thus the difference of the corresponding XMCD signals gives exclusive access to the magnetic response of layer $i=2$. In contrast to XMCD, the XAS intensity is proportional to the Ni content of the sample, and therefore independent of temperature. In order to extract additional information enabling a crosscheck of our thickness estimate, we measure at normal and grazing incidence. We note that the shape of the $\mathrm{Ni} L_{2,3}$ absorption edge cannot be used for the determination of the $\mathrm{Ni}$ content since it does not change markedly for compositions with a $\mathrm{Ni}$ content beyond $75 \%[44,45]$.

Figure 2 shows XAS and XMCD measurements performed at $3 \mathrm{~K}$ and $160 \mathrm{~K}$ sample temperature and under normal $\left(\theta=0^{\circ}\right)$ and grazing incidence $\left(\theta=70^{\circ}\right)$. We first determine the spin and orbital magnetic moments per $\mathrm{Ni}$ atom at both temperatures and incident angles from the respective XMCD signals. To very good approximation, we can neglect the dipolar term in the sum rules [46,47]. For the number of $d$ holes per Ni atom we take the Ni bulk value of $h_{d}=1.66$ [48] leading to the spin, orbital, and total moments $\mu=\mu_{L}+\mu_{S}$ reported in Table I. 


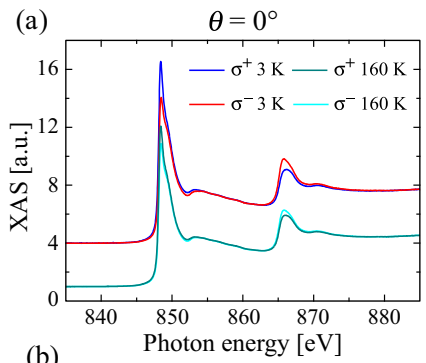

(b)

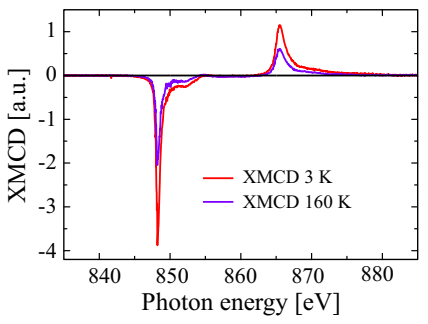

(c)

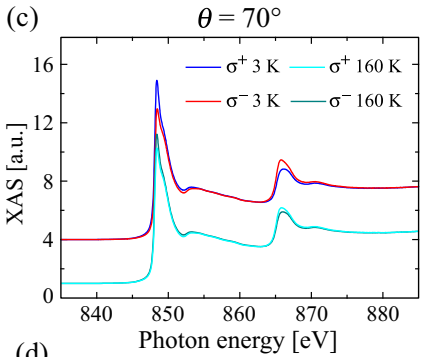

(d)
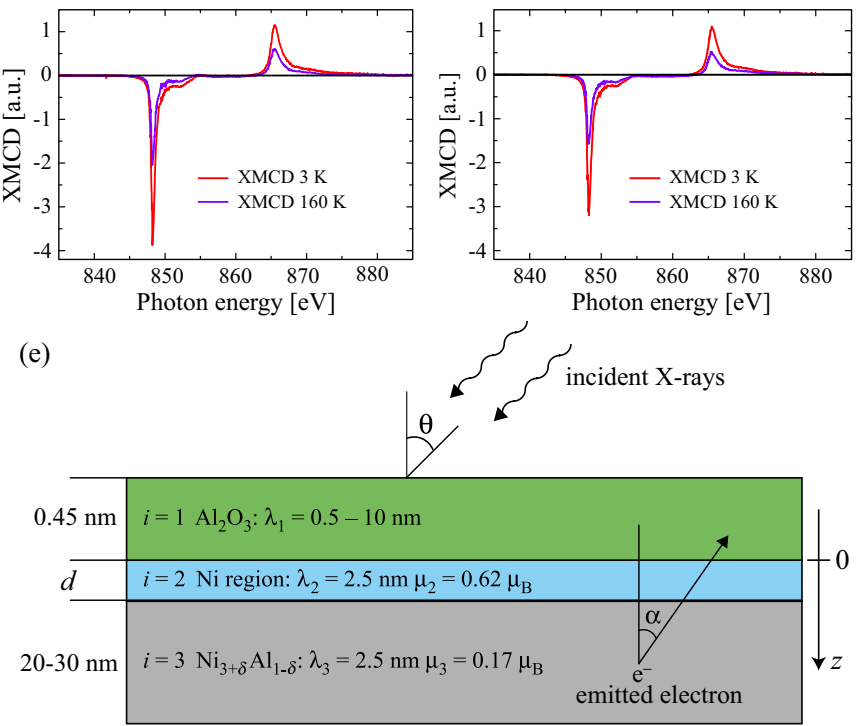

FIG. 2. (Color online) (a) Normal and (c) grazing incidence XAS at the Ni $L_{2,3}$ absorption edges ( $B=1 \mathrm{~T}, T=3$ and $160 \mathrm{~K}$ ). (b) Normal and (d) grazing incidence XMCD signals derived from (a), respectively (c). The XAS spectra acquired at different temperatures have been offset for clarity. (e) Schematic of the oxide, pure Ni cluster modeled as a pure $\mathrm{Ni}$ layer, and slightly $\mathrm{Ni}$-enriched near surface layers, labeled $i \in\{1,2,3\}$, with their respective electron escape depths $\lambda_{i}$ and total magnetic moments per atom $\mu_{i}$.

The total moments must scale with the XAS signals of the respective layers as

$$
\begin{gathered}
\mu(T=160 \mathrm{~K})=\frac{\mu_{2} X_{2}}{X_{2}+X_{3}} \\
\mu(T=3 \mathrm{~K})=\frac{\mu_{2} X_{2}+\mu_{3} X_{3}}{X_{2}+X_{3}},
\end{gathered}
$$

with $\mu_{i}$ the magnetic moments and $X_{i}$ the integrals over the XAS intensity $(845 \leqslant E \leqslant 875 \mathrm{eV})$ of the respective layer $i$.

TABLE I. Measured orbital $\mu_{L}$, spin $\mu_{S}$, and total $\mu$ magnetic moments per $\mathrm{Ni}$ atom at $3 \mathrm{~K}$ and $160 \mathrm{~K}$ for grazing and normal incidence. The errors are $\pm 7 \%$ of the reported values.

\begin{tabular}{lcccc}
\hline \hline$\theta\left(^{\circ}\right)$ & $T(\mathrm{~K})$ & $\mu_{L}\left(\mu_{\mathrm{B}}\right)$ & $\mu_{S}\left(\mu_{\mathrm{B}}\right)$ & $\mu\left(\mu_{\mathrm{B}}\right)$ \\
\hline 0 & 160 & 0.018 & 0.199 & 0.217 \\
0 & 3 & 0.027 & 0.374 & 0.401 \\
70 & 160 & 0.022 & 0.190 & 0.212 \\
70 & 3 & 0.025 & 0.358 & 0.383 \\
\hline \hline
\end{tabular}

Therefore

$$
\frac{\mu(T=160 \mathrm{~K})}{\mu(T=3 \mathrm{~K})}=\frac{\mu_{2} X_{2}}{\mu_{2} X_{2}+\mu_{3} X_{3}} .
$$

The left hand side of this equation is measured from XMCD for both incident angles. On the right hand side we take for the moment per $\mathrm{Ni}$ atom in layer 2 the bulk value of $\mu_{2}=0.62 \mu_{\mathrm{B}}$ [49]. For $\mu$ in the $\mathrm{Ni}_{3+\delta} \mathrm{Al}_{1-\delta}$ layer, we assume a linear evolution with $\mathrm{Ni}$ content. Extrapolating from the reported values of $0.077 \mu_{\mathrm{B}}$ for $\delta=0.00$ and $0.125 \mu_{\mathrm{B}}$ for $\delta=0.04[22,23]$, we arrive for our case of $\delta=0.08$ at $\mu_{3}=0.173 \mu_{\mathrm{B}}$.

The incident angle and the layer thicknesses enter the expressions of $X_{i}$. To very good approximation, we can neglect the $\mathrm{Al}$ absorption and assume the XAS integral to be proportional to the XAS intensity at the Ni $L_{3}$ edge. Due to the long X-ray penetration depth of $\lambda=400 \mathrm{~nm} \mathrm{[43]} \mathrm{it} \mathrm{is}$ justified to assume equal incident photon flux for layers 2 and 3. Assuming further equal absorption cross sections for both layers, one obtains $[43,50,51]$

$$
\begin{gathered}
X_{2} \propto \int_{-\pi / 2}^{\pi / 2} \int_{0}^{d} e^{\frac{-(z+l)}{\lambda^{\cos \theta}}} e^{\frac{-l}{\lambda_{1} \cos \alpha}} e^{\frac{-z}{\lambda_{2} \cos \alpha}} d z d \alpha \\
X_{3} \propto \frac{3+\delta}{4} \int_{-\pi / 2}^{\pi / 2} \int_{d}^{\infty} e^{\frac{-(z+l)}{\lambda^{2} \cos \theta}} e^{\frac{-l}{\lambda_{1} \cos \alpha}} e^{\frac{-d}{\lambda_{2} \cos \alpha}} e^{\frac{-z+d}{\lambda_{3} \cos \alpha}} d z d \alpha,
\end{gathered}
$$

with $d, l, \delta, \theta$, and $\lambda$ defined above, $\lambda_{i}$ the electron escape depths of layer $i$, and $\alpha$ the emitted electron angle with respect to the surface normal.

The electrons' escape depths are critical parameters in our estimation. The TEY signal is dominated by the secondary electrons generated from the inelastic cascade process of the high energy primary Auger electrons created by the core-hole decay $[43,52]$. This leads to an energy dependence of $\lambda_{2}$ and $\lambda_{3}$. We use $\lambda_{2}=2.5 \mathrm{~nm}$ measured for Ni bulk in similar XMCD experiments [43] and assume an equal value for $\lambda_{3}$. Since $\lambda_{1}$ is less known, we calculated our thickness $d$ for several reasonable values of it. $\lambda_{1}=0.5,1.0$, and $10 \mathrm{~nm}$ yield $0.37 \leqslant$ $d \leqslant 0.64 \mathrm{~nm}, 0.35 \leqslant d \leqslant 0.61 \mathrm{~nm}$, and $0.30 \leqslant d \leqslant 0.55 \mathrm{~nm}$, respectively. The error bars are essentially caused by the errors in $\mu_{2}$ and $\mu_{3}$, see caption of Table I. However, for a given choice of $\mu_{2}$ and $\mu_{3}$, the thicknesses inferred from our normal and grazing incidence measurements agree within $0.02 \mathrm{~nm}$. In conclusion, the equivalent thickness of pure $\mathrm{Ni}$ present in $\mathrm{Ni}$ clusters at the alumina/alloy interface is $d=0.45 \pm 0.15 \mathrm{~nm}$ and therefore around two Ni monolayers.

\section{Magnetism of self-assembled Fe nanoclusters}

Figures 3(a) and 3(b) compare Fe nanoclusters grown with and without Pd predeposition. It is evident that the Pd-seeded Fe nanoclusters are much more regularly spaced and more monodisperse than the ones grown without [14]. The mean size of the Pd-seeded Fe clusters, as inferred from the respective coverages, is 308 atoms with $241 \mathrm{Fe}$ and $67 \mathrm{Pd}$ atoms. Note that the clusters are three dimensional in both cases.

The temperature-dependent out-of-plane zero-field susceptibilities of the two systems are compared in Fig. 3(c) with that of the pristine substrate. The different intensities of the plateaus between $T_{\mathrm{C}_{1}}$ and $T_{\mathrm{C}_{2}}$ are within the variation observed 
(a)

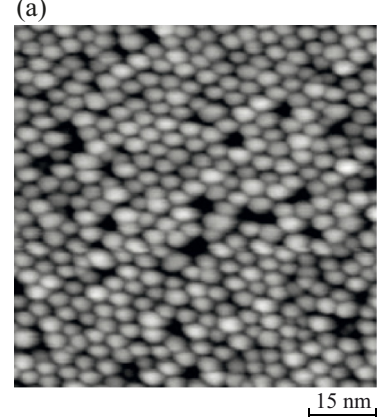

(c)

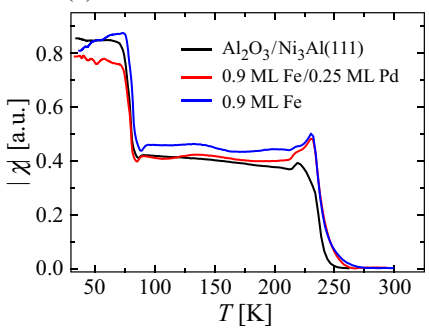

(b)

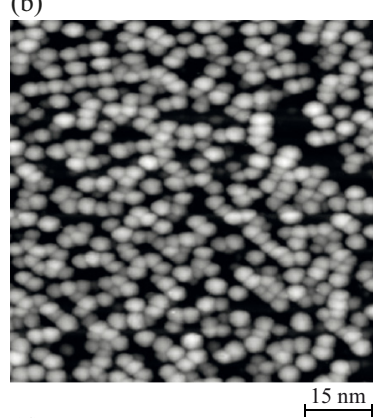

(d) (a)

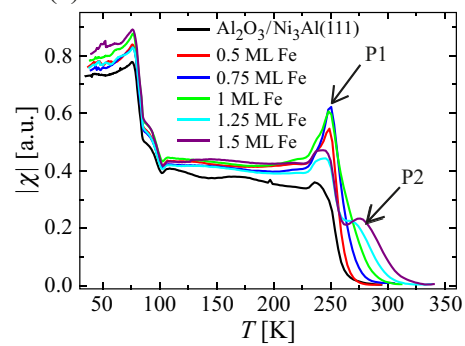

(c)

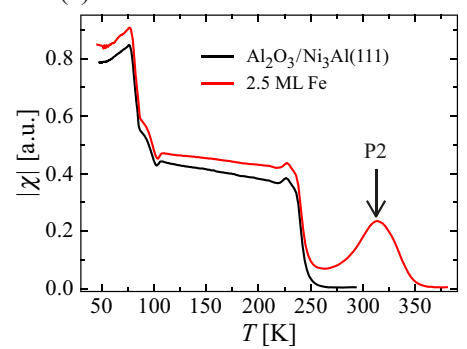

(e)

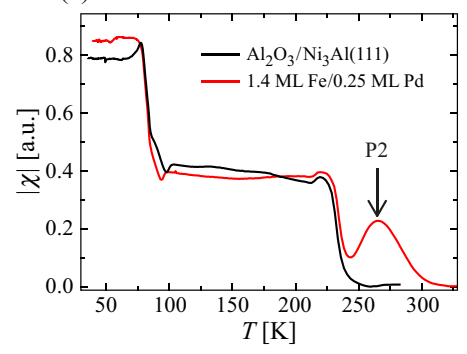

(b)

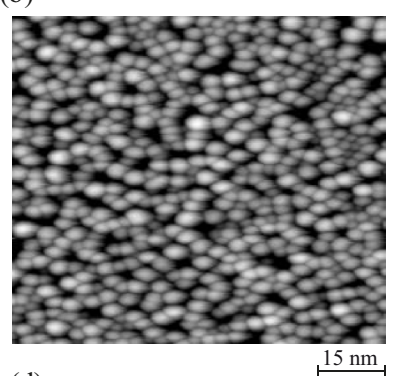

(d)

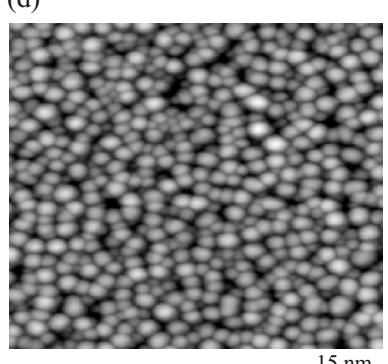

$15 \mathrm{~nm}$

FIG. 3. (Color online) STM images of 0.9 ML Fe (a) deposited after 0.25 ML Pd have been deposited for cluster seeding at the corner holes of the $\mathrm{Al}_{2} \mathrm{O}_{3} / \mathrm{Ni}_{3} \mathrm{Al}(111)-(\sqrt{67} \times \sqrt{67}) \mathrm{R} 12.2^{\circ}$ surface and (b) without seeding. (c) MOKE out-of-plane $\chi(T)$ of the substrate (black), of 0.9 ML Fe deposited on Pd-seeded (red) and unseeded (blue) alumina. (d) In-plane magnetization curves acquired for the three cases with transverse MOKE at $90 \mathrm{~K}$. The curves have been normalized to the high field values.

between different $\mathrm{Al}_{2} \mathrm{O}_{3} / \mathrm{Ni}_{3} \mathrm{Al}(111)$ samples (see Fig. 1 (d)) and not significant. The only signature of the Fe clusters is therefore a peak close to $T_{\mathrm{C}_{2}}$. Neither its shape nor height and position depend on $\mathrm{Pd}$ seeding. We attribute this peak to a magnetic coupling of the Fe clusters across the oxide to the underlying substrate. Since the peak is located close to the critical temperature of the $\mathrm{Ni}$ interface region, the surface clusters couple to that region. This assignment is further supported by the in-plane magnetization curves recorded with MOKE at $90 \mathrm{~K}$, where that region is ferromagnetic with in-plane easy axis. The curves are shown in Fig. 3(d); they have identical coercive fields for the three systems indicating that the Fe clusters are firmly coupled to the Ni interface region. The peak results from the fact that this region undergoes a phase transition from ferro- to paramagnetic at $T_{\mathrm{C}_{2}}$. From there on, the Fe clusters become decoupled and display their intrinsic magnetism. This can either be superparamagnetism with their macrospin being for $T>T_{\mathrm{C}_{2}}$ free to align with the external field, or they undergo themselves a phase transition from ferroto paramagnetic at a slightly higher temperature. In the first case the peak maximum would mark the blocking temperature $T_{\mathrm{b}}$, while in the second case it would indicate the clusters' Curie temperature $T_{\mathrm{C}}$.

In order to discriminate between the two scenarios, we performed susceptibility and STM measurements as a function of the Fe coverage on the unseeded surface, see Figs. 4(a)-4(d). Clearly, for coverages below $1 \mathrm{ML}, \chi(T)$ exhibits a single Fe cluster-related peak (P1) located at $T_{\mathrm{C}_{2}}$, whereas a high$T$ shoulder evolves at 1.25 ML. At 1.5 ML, this shoulder becomes a clear second peak (P2), and simultaneously P1

FIG. 4. (Color online) (a) MOKE zero-field out-of-plane magnetic susceptibility $\chi(T)$ of $\mathrm{Fe}$ clusters deposited on the $\mathrm{Al}_{2} \mathrm{O}_{3} / \mathrm{Ni}_{3} \mathrm{Al}(111)$ substrate as a function of Fe coverage. (b) STM image of 1.5 ML Fe clusters. (c) $\chi(T)$ of $2.5 \mathrm{ML}$ Fe clusters (red) compared with bare substrate (black). (d) STM image of 2.5 ML Fe clusters. (e) $\chi(T)$ of 1.4 ML Fe clusters seeded on 0.25 ML Pd on $\mathrm{Al}_{2} \mathrm{O}_{3} / \mathrm{Ni}_{3} \mathrm{Al}(111)$ (red) compared with bare $\mathrm{Al}_{2} \mathrm{O}_{3} / \mathrm{Ni}_{3} \mathrm{Al}(111)$ (black). (f) STM image of 1.4 ML Fe clusters seeded on 0.25 ML Pd.

reduces its intensity. At larger Fe coverages, P2 is completely separated from the substrate magnetic phase transition and thus its intrinsic shape is observed. For example, for $2.5 \mathrm{ML}$ Fe, P2 is located at $T=315 \mathrm{~K}$ [see Fig. 4(c)]. The same behavior is observed for Fe clusters grown on the Pd-seeded alumina surface [see Fig. 4(e)]. The steep decrease of the $\chi(T)$ signal after P2 strongly suggests that we are observing the Curie temperature of the clusters; if it were a transition from blocking to superparamagnetic, the $\chi(T)$ decrease would have been much more shallow $[20,30]$. That the peak maximum indeed marks the clusters $T_{\mathrm{C}}$ is confirmed by the shape of the out-of-plane magnetization curves measured at temperatures close to P2 for large Fe coverages on both seeded and unseeded clusters [Figs. 5(a) and 5(b)] and by the Arrott plots derived from them [Figs. 5(c) and 5(d)] [53,54]. As expected, the magnetization curves show a square shape which becomes an S shape approaching P2 and then flatten quickly as the temperature is raised above $\mathrm{P} 2$ [54].

The shape of the magnetization curves also demonstrates that the Fe clusters' easy magnetization axes switch from 
(a)

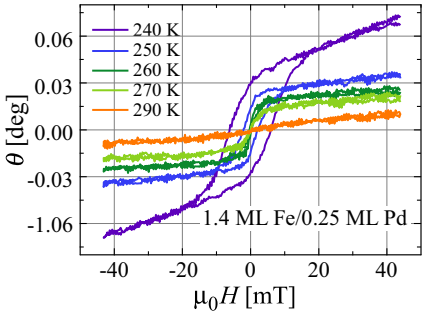

(c)

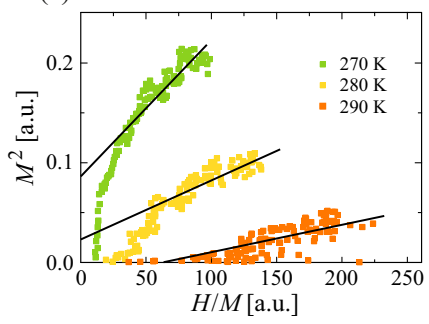

(b)

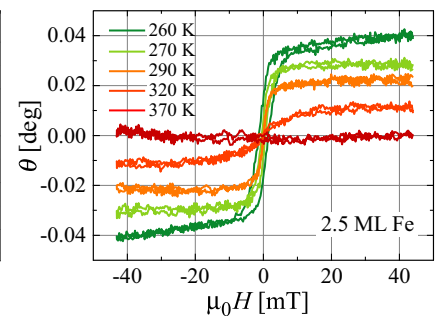

(d)

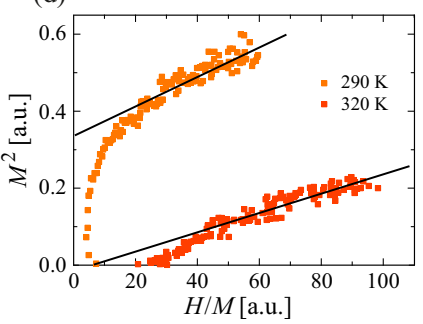

FIG. 5. (Color online) (a) Temperature-dependent out-of-plane magnetization curves reported in Kerr rotation angles $\theta$ for 1.4 ML Fe clusters seeded on $0.25 \mathrm{ML} \mathrm{Pd}$ on $\mathrm{Al}_{2} \mathrm{O}_{3} / \mathrm{Ni}_{3} \mathrm{Al}(111)$. (b) Same as (a) but for unseeded 2.5 ML Fe clusters. (c) Arrott plot from (a) used to infer the critical temperature of $T_{\mathrm{C}}=285 \pm 5 \mathrm{~K}$. (d) Arrott plot derived from (b) yielding $T_{\mathrm{C}}=320 \pm 5 \mathrm{~K}$ for unseeded 2.5 ML Fe clusters.

in-plane to out-of-plane above $T_{\mathrm{C}_{2}}$. The Arrott plots determine $T_{C}$ as that temperature where the linearly extrapolated highfield data of the $M^{2}(H / M)$ curves intercept the origin [solid lines in Figs. 5(c) and 5(d)] [53,54]. We derive $T_{\mathrm{C}}=285 \pm 5 \mathrm{~K}$ for 1.4 ML Fe on the Pd-seeded surface, which corresponds to clusters with a mean size of 440 atoms, and $T_{\mathrm{C}}=320 \pm 5 \mathrm{~K}$ for unseeded 2.5 ML Fe clusters.

In the absence of structural rearrangements, the $T_{\mathrm{C}}$ of nanostructures evolves monotonically with size. For Fe coverages smaller than 1.0 ML we extrapolate from the above values that $T_{\mathrm{C}} \leqslant 260 \mathrm{~K}$. We therefore attribute the sharp P1 peak to a coincidence between the Curie temperatures of the $\mathrm{Ni}$ interface region and of the Fe clusters. Note that the clusters' $T_{\mathrm{C}}$ increases only slowly with Fe coverage. In addition, at 2.5 ML we might well have reached the cluster coalescence threshold, especially on the unseeded alumina surface. However, there are still clear gaps observable between the nanoparticles in the STM images presented in Figs. 4(b), 4(d), and 4(f) suggesting cluster coalescence to be restricted to their basis. It has been shown that this effect leads to a slow enhancement of $T_{\mathrm{C}}$ [55].

\section{Nature and strength of the magnetic coupling to the $\mathrm{Ni}$ interface region}

The data presented so far point to the existence of magnetic coupling between Fe clusters and substrate, though the presence of Fe does not change the susceptibility below $T_{\mathrm{C}_{2}}$. This is surprising, since the atomic magnetic moment of $\mathrm{Fe}$ is typically four times larger than the one of $\mathrm{Ni}$ (0.62 and $2.22 \mu_{\mathrm{B}}$, respectively [49]), hence $2.5 \mathrm{ML}$ of Fe should produce a strong change in $\chi$. In particular, a ferromagnetic (antiferromagnetic) coupling between the $\mathrm{Fe}$ clusters and the $\mathrm{Ni}$ interface region should increase (decrease) the plateau heights well beyond our sensitivity. We propose

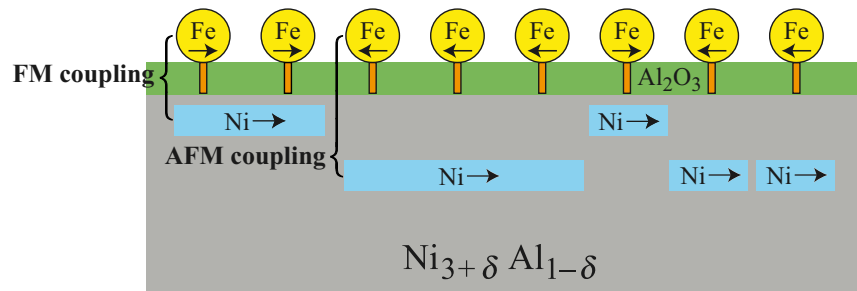

FIG. 6. (Color online) Schematic cross section of the sample. The Ni-rich interface layer is made of Ni clusters embedded in the $\mathrm{Ni}_{3} \mathrm{Al}$ sample. Their distance from the surface varies between clusters implying a different sign of the magnetic exchange coupling between them and the surface adsorbed Fe clusters. The arrows denote the magnetization direction of $\mathrm{Fe}$ and $\mathrm{Ni}$.

that the Fe cluster-surface coupling locally varies between antiferromagnetic (AFM) and ferromagnetic (FM), and this in a way that the average contribution to the total susceptibility cancels out.

We consider two coupling mechanisms, namely dipolar and exchangelike interactions. In our geometry, dipolar interactions induce an antiferromagnetic coupling independent of distance. Therefore a pure dipolar interaction cannot account for our results. Instead, for an exchangelike coupling through the alumina thin film, or through metal atoms that have created bridges through the corner holes of the $\mathrm{Al}_{2} \mathrm{O}_{3}$ layer, we expect the distance between the $\mathrm{Ni}$ interface region and the Fe clusters to define the sign of the exchange coupling. In multilayers made of magnetic films separated by nonmagnetic metallic spacers, the coupling oscillates from AFM to FM with a spacer thickness variation of only a few $\AA$ [56-58]. In the case of insulating spacers, one has to distinguish two thickness regimes. For large thicknesses, the evolution of the coupling constant with spacer thickness is monotonic without sign change [59], whereas for a few $\AA$ thick oxide spacers, which is the case in the present system, a sign change has been reported for the magnetic exchange energy [60]. Therefore independent of the existence of metal bridges or coupling through the oxide, the sign of the coupling is expected to change with the distance between the ferromagnetic materials. Assuming that the $\mathrm{Ni}$ interface region is made of Ni clusters embedded in the $\mathrm{Ni}_{3+\delta} \mathrm{Al}_{1-\delta}$ matrix, their distance from the interface can differ from place to place (see Fig. 6). Thus, the coupling between the Fe clusters and the embedded $\mathrm{Ni}$ clusters can also change from AFM to FM depending on the cluster location on the surface. In zero-field susceptibility measurements these two contributions have opposite signs, leading to unchanged intensity for the plateaus. Note that the Ni cluster size can't be determined in our experiment; we can only determine the $T_{\mathrm{C}}$.

Within our model, the Fe clusters are coupled to the substrate up to $T_{\mathrm{C}_{2}}$, where the substrate becomes paramagnetic. The susceptibility measurements can then be interpreted in the framework proposed by Won et al. [61]. They have shown that two ferromagnetically coupled layers give rise to a resonant peak in susceptibility if their Curie temperatures are close to one another. Moreover, the Curie temperature of the coupled system is slightly increased with respect to the uncoupled case. In our case, the resonating peaks appear for $\mathrm{Fe}$ coverages in 


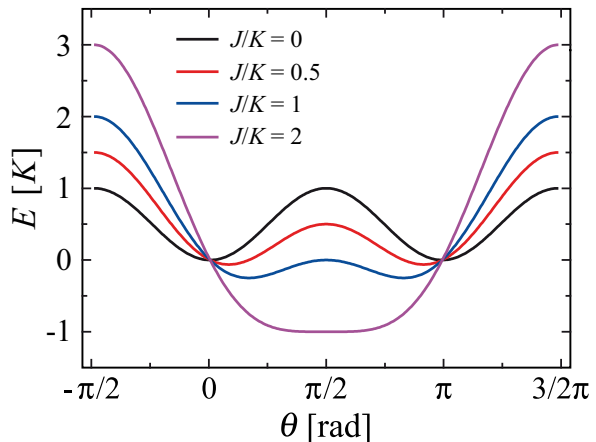

FIG. 7. (Color online) Energy profiles as a function of the angle between the magnetization and the surface normal derived from Eq. (7) for different $J / K$. The condition $J \geqslant 2 K$ is required to have an in-plane stable minimum.

between 0.5 and $1 \mathrm{ML}$ on the bare alumina surface. Figure 4(a) shows a slight increase of the temperature of $\mathrm{P} 1$ when the Fe coverage increases from 0.5 to $1 \mathrm{ML}$, while $T_{\mathrm{C}_{2}}$ recovers the value observed on the bare alumina for $1.5 \mathrm{ML}$. At this coverage and above, the Fe clusters magnetization switches to out-of-plane for $T>T_{\mathrm{C}_{2}}$ due to the vanishing of the coupling with the substrate, and the peak position of $\mathrm{P} 2$ represents the intrinsic Curie temperature of the Fe clusters. For coverages below $0.5 \mathrm{ML}$ we were not able to observe any $\mathrm{Fe}$ related feature in $\chi(T)$. This is explained by the fact that very small Fe clusters present at these low coverages have small $M$ values with respect to the $\mathrm{Ni}$ interface region. In addition, they have $T_{C} \ll T_{\mathrm{C}_{2}}$, and therefore resonance effects enhancing the signal are not expected.

An estimation of the coupling strength can be derived from the following considerations. Due to the out-of-plane anisotropy for the Fe clusters [see Figs. 5(a) and 5(b)], the coupling has to be strong enough to block the Fe cluster magnetization in the surface plane up to $T_{\mathrm{C}_{2}}$. Considering the case where an Fe cluster has FM coupling with the substrate, the cluster energy is then given in negligible external field by

$$
E(\theta)=K \sin ^{2}(\theta)-J \cos (\pi / 2-\theta),
$$

where $K$ is the Fe cluster out-of-plane magnetic anisotropy energy, $\theta$ the angle between the cluster magnetization and the surface normal, and $J$ the cluster coupling energy to the underlying Ni interface region, the magnetization of which lies in the plane with $\theta=\pi / 2 . K$ can be expressed as the magnetic anisotropy energy per atom $K_{\text {at }}$ times the number of atoms per cluster $N$. Very likely, $J$ depends on the number of atoms at the cluster base, i.e., $J=J_{\text {at }} N_{\text {base }}$. This can be estimated to be about 88 atoms in the case of the Pd-seeded surface presented in Fig. 3(a) assuming a hemispherical clusters geometry. Figure 7 shows the energy profile for different $J / K$ ratios. It is evident that a stable in-plane minimum requires $J \geqslant 2 K$. The same requirement is found for the antiferromagnetic coupling. The case $0<J<2 K$ is unlikely since this would give two stable canted minima with an energy barrier reduced with respect to the case with $J=0$. Due to thermal excitations the Fe cluster magnetization would then switch from one stable position to the other, resulting in an increase of the zero-field susceptibility in the plateau regions that we do not observe.
A quantitative evaluation of the coupling constant from our model requires us to assume a $K$ value for our Fe clusters. The anisotropy energy strongly depends on the morphology of the $\mathrm{Fe}$ nanoclusters. Fe trimers on $\mathrm{Al}_{2} \mathrm{O}_{3} / \mathrm{Ni}_{3} \mathrm{Al}(111)$ exhibit $K_{\text {at }}=0.5 \mathrm{meV}$ [25], while this value is expected to drop quickly with the cluster size [5]. $1 \mathrm{ML}$ thick Fe films on transition metal substrates have $K_{\text {at }}=0.1 \mathrm{meV}[62,63]$. For Fe nanostructures with $2.5 \mathrm{~nm}$ mean diameter embedded in a $\mathrm{Ag}$ matrix, $K_{\text {at }}=10 \mu \mathrm{eV}$ has been reported [64]. Therefore it is reasonable to assume $K_{\text {at }}=0.1 \mathrm{meV}$ for the present system leading to $K=24 \mathrm{meV}$ per cluster in the case of $0.9 \mathrm{ML} \mathrm{Fe}$ grown with Pd seeding on the alumina surface [Fig. 3(a)]. This requires a coupling energy of $J=48 \mathrm{meV}$. With 88 atoms at the cluster base we derive $J_{\text {at }}=0.55 \mathrm{meV}$. Similar values have been reported for interlayer exchange coupled systems $[58,60,65]$.

\section{SUMMARY}

We have characterized the magnetic properties of $\mathrm{Fe}$ nanocluster superlattices grown on 2 ML thick alumina on $\mathrm{Ni}_{3} \mathrm{Al}(111)$ forming a $(\sqrt{67} \times \sqrt{67}) \mathrm{R} 12.2^{\circ}$ unit cell. The substrate itself has intriguing magnetic properties. Namely, it has a ferromagnetic slightly $\mathrm{Ni}$-enriched region, at least 20-30 nm thick, with composition $\mathrm{Ni}_{3+\delta} \mathrm{Al}_{1-\delta}, \delta=0.084$. It originates from the different sputtering yields of $\mathrm{Ni}$ and $\mathrm{Al}$, leading to an $\mathrm{Al}$ depletion during the course of the repeated preparation and also oxidation cycles. It has an in-plane easy axis and a Curie temperature that is with $T_{\mathrm{C}_{1}}=81 \pm 3 \mathrm{~K}$ twice as high as the one of the stoichiometric bulk substrate. As expected, $T_{\mathrm{C}_{1}}$ increases monotonically with the number of sample preparation and oxidation cycles. At the near surface region there is a second much thinner and more strongly $\mathrm{Ni}$ enriched layer that has $T_{\mathrm{C}_{2}}=240 \pm 12 \mathrm{~K}$. This layer has again an in-plane easy magnetization axis, and it exists for the bare and for the oxide covered surface.

The Fe cluster growth is well ordered when seeded on predeposited $\mathrm{Pd}$, whereas the clusters are more randomly spaced and also more polydisperse without this seeding. The magnetic properties of the Fe clusters appear to be dominated for $T<T_{\mathrm{C}_{2}}$ by their coupling to the underlying substrate. We propose a near surface layer with Ni clusters embedded in a $\mathrm{Ni}_{3+\delta} \mathrm{Al}_{1-\delta}$ matrix. We further propose that the $\mathrm{Fe}$ clusters couple to the buried $\mathrm{Ni}$ clusters by exchange, or superexchange, through the oxide and that the coupling must have a strength of at least two times the anisotropy energy. In addition, this coupling has to alternate from antiferro- to ferromagnetic between different surface areas, since the cluster sample shows no increase in magnetic susceptibility with respect to the bare oxide surface. We explain this alternation by the different distances of the embedded Ni clusters from the oxide/substrate interface.

The intrinsic cluster properties can be accessed for $T>T_{\mathrm{C}_{2}}$, where this coupling vanishes. This is only possible for cluster sizes from 440 atoms on for which we find $T_{\mathrm{C}}>T_{\mathrm{C}_{2}}$. The easy magnetization axis of these Fe clusters is out-of-plane, and the Curie temperature increases monotonically with size. None of the magnetic properties depend on whether or not the clusters were seeded by Pd. 
[1] H. Brune, M. Giovannini, K. Bromann, and K. Kern, Nature (London) 394, 451 (1998).

[2] H. Brune, in Properties of Single Organic Molecules on Crystal Surfaces, edited by F. Rosei, P. Grütter, and W. Hofer (Springer, New York, 2006), p. 247.

[3] U. Heiz, S. Abbet, A. Sanchez, and W. D. Schneider, J. Am. Chem. Soc. 121, 3214 (1999).

[4] G. Pacchioni and H. Freund, Chem. Rev. 113, 4035 (2013).

[5] P. Gambardella, S. Rusponi, M. Veronese, S. S. Dhesi, C. Grazioli, A. Dallmeyer, I. Cabria, R. Zeller, P. H. Dederichs, K. Kern, C. Carbone, and H. Brune, Science 300, 1130 (2003).

[6] H. Brune and P. Gambardella, Surf. Sci. 603, 1812 (2009).

[7] B. Voigtländer, G. Meyer, and N. M. Amer, Phys. Rev. B 44, 10354 (1991).

[8] L. Vitali, M. G. Ramsey, and F. P. Netzer, Phys. Rev. Lett. 83, 316 (1999).

[9] H. Ellmer, V. Repain, M. Sotto, and S. Rousset, Surf. Sci. 511, 183 (2002).

[10] S. Rousset, V. Repain, G. Baudot, H. Ellmer, Y. Garreau, V. Etgens, J. M. Berroir, B. Croset, M. Sotto, P. Zeppenfeld, J. Ferré, J. P. Jamet, C. Chappert, and J. Lecoeur, Mat. Sci. Eng. B 96, 169 (2002).

[11] J. L. Li, J. F. Jia, X. J. Liang, X. Liu, J. Z. Wang, Q. K. Xue, Z. Q. Li, J. S. Tse, Z. Zhang, and S. B. Zhang, Phys. Rev. Lett. 88, 066101 (2002).

[12] F. Leroy, G. Renaud, A. Letoublon, R. Lazzari, C. Mottet, and J. Goniakowski, Phys. Rev. Lett. 95, 185501 (2005).

[13] A. T. N'Diaye, T. Gerber, C. Busse, J. Mysliveček, J. Coraux, and T. Michely, New J. Phys. 11, 103045 (2009).

[14] M. Schmid, G. Kresse, A. Buchsbaum, E. Napetschnig, S. Gritschneder, M. Reichling, and P. Varga, Phys. Rev. Lett. 99, 196104 (2007).

[15] S. Degen, C. Becker, and K. Wandelt, Faraday Discuss. 125, 343 (2004).

[16] C. Becker, A. Rosenhahn, A. Wiltner, K. von Bergmann, J. Schneider, P. Pervan, M. Milun, M. Kralj, and K. Wandelt, New J. Phys. 4, 75 (2002).

[17] G. Hamm, C. Becker, and C. R. Henry, Nanotechnol. 17, 1943 (2006).

[18] A. Buchsbaum, M. De Santis, H. C. N. Tolentino, M. Schmid, and P. Varga, Phys. Rev. B 81, 115420 (2010).

[19] L. Gragnaniello, T. Ma, G. Barcaro, L. Sementa, F. R. Negreiros, A. Fortunelli, S. Surnev, and F. P. Netzer, Phys. Rev. Lett. 108, 195507 (2012).

[20] N. Weiss, T. Cren, M. Epple, S. Rusponi, G. Baudot, S. Rohart, A. Tejeda, V. Repain, S. Rousset, P. Ohresser, F. Scheurer, P. Bencok, and H. Brune, Phys. Rev. Lett. 95, 157204 (2005).

[21] S. Rohart, V. Repain, A. Tejeda, P. Ohresser, F. Scheurer, P. Bencok, J. Ferré, and S. Rousset, Phys. Rev. B 73, 165412 (2006).

[22] F. R. de Boer, C. J. Schinkel, J. Biesterbos, and S. Proost, J. Appl. Phys. 40, 1049 (1969).

[23] S. K. Dhar, K. A. Gschneidner, L. L. Miller, and D. C. Johnston, Phys. Rev. B 40, 11488 (1989).

[24] J. Y. Rhee, Y. V. Kudryavtsev, and Y. P. Lee, Phys. Rev. B 68, 045104 (2003).

[25] A. Lehnert, S. Rusponi, M. Etzkorn, S. Ouazi, P. Thakur, and H. Brune, Phys. Rev. B 81, 104430 (2010).

[26] E. Vesselli, A. Baraldi, S. Lizzit, and G. Comelli, Phys. Rev. Lett. 105, 046102 (2010).
[27] S. Degen, A. Krupski, M. Kralj, A. Langner, C. Becker, M. Sokolowski, and K. Wandelt, Surf. Sci. 576, L57 (2005).

[28] G. Hamm, C. Barth, C. Becker, K. Wandelt, and C. R. Henry, Phys. Rev. Lett. 97, 126106 (2006).

[29] H. Brune, H. Röder, K. Bromann, and K. Kern, Thin Solid Films 264, 230 (1995).

[30] S. Rusponi, T. Cren, N. Weiss, M. Epple, P. Buluschek, L. Claude, and H. Brune, Nat. Mater. 2, 546 (2003).

[31] C. Piamonteze, U. Flechsig, S. Rusponi, J. Dreiser, J. Heidler, M. Schmidt, R. Wetter, M. Calvi, T. Schmidt, H. Pruchova, J. Krempasky, C. Quitmann, H. Brune, and F. Nolting, J. Synchrotron Rad. 19, 661 (2012).

[32] Z. Qiu and S. Bader, J. Magn. Magn. Mater. 200, 664 (1999).

[33] D. A. Allwood, G. Xiong, M. D. Cooke, and R. P. Cowburn, J. Phys. D: Appl. Phys. 36, 2175 (2003).

[34] A. Lehnert, P. Buluschek, N. Weiss, J. Giesecke, M. Treier, S. Rusponi, and H. Brune, Rev. Sci. Instrum. 80, 023902 (2009).

[35] E. R. Moog, C. Liu, S. D. Bader, and J. Zak, Phys. Rev. B 39, 6949 (1989).

[36] Z. Q. Qiu and S. D. Bader, Rev. Sci. Instrum. 71, 1243 (2000).

[37] F. Huang, M. T. Kief, G. J. Mankey, and R. F. Willis, Phys. Rev. B 49, 3962 (1994).

[38] D. Sondericker, F. Jona, and P. M. Marcus, Phys. Rev. B 34, 6770 (1986).

[39] O. Bikondoa, G. R. Castro, X. Torrelles, F. Wendler, and W. Moritz, Phys. Rev. B 72, 195430 (2005).

[40] S. Hofmann and M. Stepanova, Appl. Surf. Sci. 90, 227 (1995).

[41] M. Abbate, J. B. Goedkoop, F. M. F. DeGroot, M. Grioni, J. C. Fuggle, S. Hofmann, H. Petersen, and M. Sacchi, Surf. Interf. Anal. 18, 65 (1992).

[42] B. H. Frazer, B. Gilbert, B. R. Sonderegger, and G. D. Stasio, Surf. Sci. 537, 161 (2003).

[43] R. Nakajima, J. Stöhr, and Y. U. Idzerda, Phys. Rev. B 59, 6421 (1999).

[44] D. A. Muller, S. Subramanian, P. E. Batson, S. L. Sass, and J. Silcox, Phys. Rev. Lett. 75, 4744 (1995).

[45] Y. Chang, K. Lin, W. Pong, M. Tsai, H. Hseih, J. Pieh, P. Tseng, J. Lee, and L. Hsu, J. Appl. Phys. 87, 1312 (2000).

[46] B. T. Thole, P. Carra, F. Sette, and G. van der Laan, Phys. Rev. Lett. 68, 1943 (1992).

[47] P. Carra, B. T. Thole, M. Altarelli, and X. Wang, Phys. Rev. Lett. 70, 694 (1993).

[48] R. Wu and A. J. Freeman, Phys. Rev. Lett. 73, 1994 (1994).

[49] J. Stöhr and H. C. Siegmann, Magnetism From Fundamentals to Nanoscale Dynamics (Springer-Verlag, Berlin, Heidelberg, 2006).

[50] R. W. Paynter, Surf. Interf. Anal. 27, 103 (1999).

[51] S. Mróz and A. Mróz, Thin Solid Films 367, 126 (2000).

[52] J. Stöhr, C. Noguera, and T. Kendelewicz, Phys. Rev. B 30, 5571 (1984).

[53] A. Arrott, Phys. Rev. 108, 1394 (1957).

[54] P. Poulopoulos, M. Farle, U. Bovensiepen, and K. Baberschke, Phys. Rev. B 55, R11961 (1997).

[55] W.-C. Lin, P.-C. Huang, K.-J. Song, and M.-T. Lin, Appl. Phys. Lett. 88, 153117 (2006).

[56] S. S. P. Parkin, N. More, and K. P. Roche, Phys. Rev. Lett. 64, 2304 (1990).

[57] P. Bruno, Phys. Rev. B 52, 411 (1995).

[58] S. O. Demokritov, J. Phys. D: Appl. Phys. 31, 925 (1998).

[59] P. Bruno, Phys. Rev. B 49, 13231 (1994). 
[60] J. Faure-Vincent, C. Tiusan, C. Bellouard, E. Popova, M. Hehn, F. Montaigne, and A. Schuhl, Phys. Rev. Lett. 89, 107206 (2002).

[61] C. Won, Y. Z. Wu, A. Scholl, A. Doran, N. Kurahashi, H. W. Zhao, and Z. Q. Qiu, Phys. Rev. Lett. 91, 147202 (2003).

[62] G. Moulas, A. Lehnert, S. Rusponi, J. Zabloudil, C. Etz, S. Ouazi, M. Etzkorn, P. Bencok, P. Gambardella, P. Weinberger, and H. Brune, Phys. Rev. B 78, 214424 (2008).
[63] A. Lehnert, S. Dennler, P. Błoński, S. Rusponi, M. Etzkorn, G. Moulas, P. Bencok, P. Gambardella, H. Brune, and J. Hafner, Phys. Rev. B 82, 094409 (2010).

[64] J. Bansmann, S. Baker, C. Binns, J. Blackman, J.-P. Bucher, J. Dorantes-Dvila, V. Dupuis, L. Favre, D. Kechrakos, A. Kleibert, K.-H. Meiwes-Broer, G. Pastor, A. Perez, O. Toulemonde, K. Trohidou, J. Tuaillon, and Y. Xie, Surf. Sci. Rep. 56, 189 (2005).

[65] Z. Y. Liu and S. Adenwalla, Phys. Rev. Lett. 91, 037207 (2003). 